\title{
Transcription Factor EB Is Selectively Reduced in the Nuclear Fractions of Alzheimer's and Amyotrophic Lateral Sclerosis Brains
}

\author{
Hongjie Wang, ${ }^{1}$ Ruizhi Wang, ${ }^{1}$ Shaohua $X u^{2}{ }^{2}$ and Madepalli K. Lakshmana ${ }^{1}$ \\ ${ }^{1}$ Section of Neurobiology, Torrey Pines Institute for Molecular Studies, 11350 SW Village Parkway, Port St. Lucie, FL 34987, USA \\ ${ }^{2}$ Florida Institute of Technology, 150 West University Boulevard, Melbourne, FL 32901, USA
}

Correspondence should be addressed to Madepalli K. Lakshmana; mlakshmana@tpims.org

Received 20 April 2016; Revised 3 June 2016; Accepted 7 June 2016

Academic Editor: Inga Kadish

Copyright (C) 2016 Hongjie Wang et al. This is an open access article distributed under the Creative Commons Attribution License, which permits unrestricted use, distribution, and reproduction in any medium, provided the original work is properly cited.

\begin{abstract}
Multiple studies suggest that autophagy is strongly dysregulated in Alzheimer's disease (AD) and amyotrophic lateral sclerosis (ALS), as evidenced by accumulation of numerous autophagosomes, lysosomes with discontinuous membranes, and aggregated proteins in the patients' brains. Transcription factor EB (TFEB) was recently discovered to be a master regulator of lysosome biogenesis and autophagy. To examine whether aberrant autophagy in AD and ALS is due to alterations in TFEB expression, we systematically quantified the levels of TFEB in these brains by immunoblotting. Interestingly, cytoplasmic fractions of AD brains showed increased levels of normalized (to tubulin) TFEB only at Braak stage IV (61\%, $p<0.01)$. Most importantly, normalized (to lamin) TFEB levels in the nuclear fractions were consistently reduced starting from Braak stage IV (52\%, $p<0.01)$, stage V (67\%, $p<0.01)$, and stage VI $(85 \%, p<0.01)$ when compared to normal control (NC) brains. In the ALS brains also, nuclear TFEB levels were reduced by $62 \%(p<0.001)$. These data suggest that nuclear TFEB is selectively lost in ALS as well as AD brains, in which TFEB reduction was Braak-stage-dependent. Taken together, the observed reductions in TFEB protein levels may be responsible for the widely reported autophagy defects in these disorders.
\end{abstract}

\section{Introduction}

Alzheimer's disease (AD) is an irreversible neurodegenerative disorder that presents with progressive intellectual deterioration involving memory, language, and judgment ultimately leading to total dependence on nursing care. It is now estimated that nearly 35.6 million patients are affected by $\mathrm{AD}$ worldwide and that about 4.6 million new cases are added each year causing enormous societal and economic burden [1]. Pathologically, AD is characterized by intracellular tau inclusions resulting from tau mutations, extracellular amyloid plaques made up of amyloid $\beta$ peptide $(\mathrm{A} \beta)$ derived from amyloid precursor protein (APP), loss of neurons and synapses, astrogliosis, microglial activation, and inflammation [2,3]. But, as of today, there is no effective therapy for $\mathrm{AD}$ and the available treatments can neither reverse nor slow the disease progression as they are not designed to treat the underlying cause of $\mathrm{AD}$. AD has been suggested to have a strong genetic basis with heritability estimates of up to $80 \%$ [4]. However, genetic variants in the four well-established genes, namely, APP, presenilin (PS) 1 , PS2, and ApoE, and the newly identified nine genetic risk factors for the late-onset $\mathrm{AD}$ (LOAD) all together account for less than half of this heritability [5]. Therefore, additional risk genes that contribute to Alzheimer's pathogenesis need to be identified.

Amyotrophic lateral sclerosis (ALS) is a fatal neurodegenerative disorder characterized by muscle wasting and paralysis due to the degeneration of lower and upper motor neurons and their axonal tracts [6]. ALS is usually fatal within 3 to 5 years following diagnosis [7]. About 90\% of ALS cases are sporadic (SALS) and the remaining about $10 \%$ are caused by mutations in more than fifteen genes and hence classified as familial (FALS) [8]. In the industrialized world, more than one in 500 people will die of the disease [9] and the societal and economic burden is substantial $[10,11]$. 
Nearly 50 randomized clinical trials (RCTs) based on diverse mechanisms have been completed, but none turned out to be successful for ALS [12]. So far, the only available drug riluzole, approved more than 20 years ago, slows the rate of progression and prolongs survival only by $2-3$ months $[13,14]$. Therefore, novel mechanisms of disease pathogenesis should be identified also for ALS.

Importantly, age is the single major risk factor for $\mathrm{AD}$, ALS, and several other neurological disorders, suggesting that there is an age-associated dysfunction of specific molecular and cellular pathways. In fact, accumulating lines of evidence suggest that autophagy, the pathway that involves delivery of cytoplasmic cargo including aggregated proteins to the lysosomes, is transcriptionally downregulated during normal aging in the human brain [15-17] and even more so in AD [18-21] and ALS [22-24]. Compounded with this deficiency, these disorders have increased production and aggregation of toxic protein aggregates due to mutations in their respective genes that invariably lead to intracytoplasmic accumulation of protein aggregates. In neurons, the two major proteolytic systems that participate in protein turnover and removal of misfolded proteins are the ubiquitin-proteasome system (UPS) and the autophagy-lysosome pathway (ALP). UPS is the first line of defense for normal unfolded protein turnover of monomers but, with the increase in size of the aggregated proteins, UPS can no longer act on them due to narrow pore size of the proteosomal barrel and under such pathological conditions autophagy becomes a vital and predominant pathway to degrade the accumulated proteins. But age-related disorders and aging itself are genetically associated with lysosomal dysfunction [25]. Accordingly, the persistent presence of aggregates that leads to irreversible neurodegeneration and clinical symptoms in these disorders suggests that autophagy response is either dysfunctional or insufficient $[26,27]$.

Recently, it was discovered that the transcription factor EB [TFEB], a basic helix-loop-helix transcription factor, is a master regulator of lysosome biogenesis [28] which also coordinates autophagy [29], thereby increasing the activity of lysosomal degradative pathways. Moreover, TFEB-induced transcription can stimulate endocytosis [30] and exocytosis [31], which additionally enhances cellular clearance to maintain neuronal proteostasis. Interestingly, TFEB activation has been shown to reduce the accumulation of the pathogenic protein in a cellular model of Huntington's disease (HD) [28] and a mouse model of Parkinson's disease (PD) [32], which was achieved by gene transfer through viral vectors. This suggests that TFEB-induced lysosome biogenesis can effectively clear protein aggregates in neurons which is expected to prevent, stop, or even reverse proteinopathy-induced neurodegeneration and associated behavioral deficits. Since autophagy is the cell's sole mechanism for the bulk degradation of organelles and long-lived proteins [33-35] and since $\mathrm{AD}$ and ALS are deficient in this function, we were interested in examining the levels of TFEB protein in the brains of pathologically confirmed cases of AD and ALS.

Here, we quantified the levels of TFEB protein by immunoblots in $\mathrm{AD}$ brains with varying degrees of pathology classified based on Braak staging and compared with those of age-matched normal controls. We found selective loss of nuclear TFEB in AD brains in a Braak-stage-dependent manner. We also confirmed reduced nuclear but not cytosolic expression of TFEB in ALS brains using TFEB-specific antibody. Interestingly, we found strong inverse correlation between the extent of pathology and loss of nuclear TFEB in AD.

\section{Materials and Methods}

2.1. Chemicals and Antibodies. Protease inhibitor cocktail (cat. \# P8340) and dithiothreitol (cat. \# D9779), sodium orthovanadate (cat. \# 450243), HEPES (cat. \# H3375), sodium chloride (cat. \# S9888), and ethylenediaminetetraacetic acid (EDTA) (cat. \# E9884) were all purchased from SigmaAldrich (St. Louis, MO, USA). Microcystin-LR (cat. \# 475815) was purchased from Calbiochem-Millipore (Temecula, CA, USA). Nonidet-P40 substitute (cat. \# M158) was obtained from Amresco (Solon, OH, USA). Polyclonal TFEB antibody (cat. \# 4240) was purchased from Cell Signaling (Danvers, MA, USA) and monoclonal TFEB antibody, clone S1 (cat. \# H00007942-M01), was purchased from Abnova (Walnut, CA, USA). Polyclonal lamin A + C antibody (cat. \# A01455) was purchased from GenScript (Piscataway, NJ, USA). Mouse monoclonal antibody against actin (cat. \# JLA20) was purchased from Developmental Studies Hybridoma Bank (DSHB), University of Iowa (Iowa City, IA, USA). Secondary antibodies such as peroxidase-conjugated AffiniPure goat anti-mouse (code \# 115-035-146) and anti-rabbit (code \# 111035-144) IgGs were purchased from Jackson ImmunoResearch Laboratories (West Grove, PA, USA).

2.2. Tissue Extraction and Immunoblotting. The AD and normal control (NC) brain tissues (hippocampus) were obtained from the "Harvard Brain Tissue Resource Center" which is supported in part by PHS grant number R24MH068855. The ALS and NC control brain tissues (motor cortex) were obtained from the NICHD Brain and Tissue Bank for Developmental Disorders at the University of Maryland, Baltimore, MD. The lysates were prepared from the $\mathrm{AD}$ and ALS brain tissues and the age-matched NC. To prepare the brain homogenates, the brain tissue was rapidly cut into small pieces on ice, weighed, and immediately taken into $1 \%$ NP40 buffer ( $50 \mathrm{mM}$ Tris- $\mathrm{HCl}, \mathrm{pH} 8.0,150 \mathrm{mM}$ $\mathrm{NaCl}, 0.02 \%$ sodium azide, $400 \mathrm{nM}$ microcystin-LR, $0.5 \mathrm{mM}$ sodium vanadate, and $1 \%$ sodium Nonidet P-40) containing complete protease inhibitor cocktail (Sigma, St. Louis, USA) at five volumes' ratio.

As TFEB is known to be present in both the nucleus and the cytosol, we separated cytosolic and nuclear fractions by following methods exactly as used previously in our laboratory [36]. Briefly, the tissues were pestle homogenized in buffer A (10 mM HEPES, pH 7.9, 10 mM NaCl, 0.1 mM EDTA, and $1 \mathrm{mM}$ dithiothreitol plus protease inhibitor cocktail) and centrifuged for $5 \mathrm{~min}$ at $3000 \mathrm{rpm}$ in cold. The supernatants were used as cytoplasmic extracts. For nuclear fractions, the pellet was dissolved in buffer C (20 mM HEPES, pH 7.9, $400 \mathrm{mM} \mathrm{NaCl}, 1 \mathrm{mM}$ EDTA, and $1 \mathrm{mM}$ dithiothreitol 
plus protease inhibitor cocktail) and vortexed vigorously for $15 \mathrm{~min}$ in the cold. The suspension was incubated for $30 \mathrm{~min}$ at $4^{\circ} \mathrm{C}$ under constant shaking. The samples were spun at $14000 \mathrm{rpm}$ for $10 \mathrm{~min}$ at $4^{\circ} \mathrm{C}$. The supernatants were diluted with buffer D (20 mM HEPES, pH 7.9, and $1 \mathrm{mM}$ EDTA plus protease inhibitor cocktail) at five final volumes and used as nuclear fractions. Protein concentrations from each sample were measured in duplicate by BCA method (Pierce Biotechnology Inc., Rockford, USA). Equal amounts of proteins were loaded into each well and subjected to SDS-PAGE. The proteins were then transferred onto PVDF membranes, blocked with 5\% milk, and incubated overnight with primary antibodies followed by one-hour incubation with HRPconjugated secondary antibodies. The protein signals were detected using Super Signal West Pico Chemiluminescent substrate (Pierce Biotechnology Inc., USA) and normalized to tubulin levels used as loading control.

2.3. Statistical Analysis. Immunoblot signals for TFEB in the $\mathrm{AD}$ and ALS brains were quantified using publicly available Java-based ImageJ software. The protein levels in NC and $\mathrm{AD}$ brains were analyzed by one-way analysis of variance (ANOVA) followed by Dunnett multiple comparisons post hoc test for comparisons among AD and NC brains. To compare between ALS and NC brains, we used Student's paired $t$-test with two-tail $p$ value using InStat 3 software (GraphPad Software, San Diego, CA, USA). The data presented are mean \pm SEM. The data were considered significant only if $p<0.05$; $* *$ indicates $p<0.01$ and $* * *$ indicates $p<0.001$.

\section{Results}

3.1. Striking Reduction of TFEB Protein Levels in the Nuclear Fractions of AD Brains. Because AD brains show severe dysregulation of autophagy [18-21] and since TFEB is the master regulator of lysosome biogenesis which is responsible for regulating autophagy $[28,29]$, we wanted to verify whether the protein levels of TFEB are altered in AD brains. Therefore, we processed and quantified TFEB protein levels in AD brains with varying degrees of pathology and compared them with age-matched normal controls. The demographics of NC and $\mathrm{AD}$ patients are given in Table 1 . The age range of NC subjects was 58-86 years and the postmortem interval (PMI) was between 24.08 and $29.18 \mathrm{~h}$. For AD patients, the age range was 71-97 years and the PMI were 6.33-30.83 h.

Although a majority of endogenous TFEB is found in the cytoplasm under basal conditions, lower levels of TFEB can also be found in the nucleus and a fraction on the lysosomes as well [37]. However, under conditions of stress such as starvation, majority of cytoplasmic TFEB translocate to the nucleus and regulate transcription [37], resulting in biogenesis of new lysosomes [28, 29]. Therefore, we quantified TFEB protein levels in the nuclear and cytosolic fractions of $\mathrm{AD}$ brains and compared them with those of $\mathrm{NC}$ brains. Positive detection of tubulin in the cytosolic fractions (Figure 1) and lamin $\mathrm{A}$ and lamin $\mathrm{C}$ in the nuclear fractions (Figure 2) ensured noncontamination of the cytosolic and nuclear preparations. In the cytosolic fractions, normalized
TABLE 1: Demographics of normal control subjects and Alzheimer's disease patients obtained from Harvard Brain Tissue Resource Center, used in this study.

\begin{tabular}{|c|c|c|c|c|c|}
\hline Numbers & Diagnosis & Age (y) & Sex & PMI (h) & Tissue \\
\hline 1 & Normal control & 58 & F & 26.60 & Hippocampus \\
\hline 2 & Normal control & 77 & F & 28.00 & Hippocampus \\
\hline 3 & Normal control & 82 & M & 24.08 & Hippocampus \\
\hline 4 & Normal control & 86 & F & 29.18 & Hippocampus \\
\hline 5 & Normal control & 82 & F & 24.42 & Hippocampus \\
\hline 6 & $\mathrm{AD} /$ Braak 2 & 72 & M & 6.83 & Hippocampus \\
\hline 7 & AD/Braak 2 & 74 & M & 25.00 & Hippocampus \\
\hline 8 & $\mathrm{AD} /$ Braak 2 & 81 & $\mathrm{~F}$ & 6.33 & Hippocampus \\
\hline 9 & AD/Braak 3 & 87 & $\mathrm{~F}$ & 22.32 & Hippocampus \\
\hline 10 & AD/Braak 3 & 97 & $\mathrm{~F}$ & 20.66 & Hippocampus \\
\hline 11 & $\mathrm{AD} /$ Braak 3 & 77 & M & 30.83 & Hippocampus \\
\hline 12 & AD/Braak 3 & 80 & M & 23.17 & Hippocampus \\
\hline 13 & $\mathrm{AD} /$ Braak 3 & 88 & $\mathrm{~F}$ & 17.67 & Hippocampus \\
\hline 14 & $\mathrm{AD} /$ Braak 3 & 89 & M & 27.17 & Hippocampus \\
\hline 15 & $\mathrm{AD} /$ Braak 4 & 90 & M & 24.00 & Hippocampus \\
\hline 16 & $\mathrm{AD} /$ Braak 4 & 84 & $\mathrm{~F}$ & 22.08 & Hippocampus \\
\hline 17 & AD/Braak 4 & 83 & $\mathrm{~F}$ & 15.63 & Hippocampus \\
\hline 18 & $\mathrm{AD} /$ Braak 4 & 81 & F & 22.00 & Hippocampus \\
\hline 19 & $\mathrm{AD} /$ Braak 4 & 93 & $\mathrm{~F}$ & 21.50 & Hippocampus \\
\hline 20 & $\mathrm{AD} /$ Braak 5 & 83 & M & 15.90 & Hippocampus \\
\hline 21 & $\mathrm{AD} /$ Braak 5 & 73 & $\mathrm{~F}$ & 29.92 & Hippocampus \\
\hline 22 & $\mathrm{AD} /$ Braak 5 & 83 & M & 8.67 & Hippocampus \\
\hline 23 & $\mathrm{AD} /$ Braak 5 & 80 & M & 27.50 & Hippocampus \\
\hline 24 & $\mathrm{AD} /$ Braak 5 & 80 & F & 9.42 & Hippocampus \\
\hline 25 & AD/Braak 6 & 71 & M & 16.62 & Hippocampus \\
\hline 26 & AD/Braak 6 & 84 & M & 6.66 & Hippocampus \\
\hline 27 & AD/Braak 6 & 95 & M & 15.00 & Hippocampus \\
\hline 28 & AD/Braak 6 & 72 & M & 16.08 & Hippocampus \\
\hline
\end{tabular}

AD: Alzheimer's disease; M: male; F: female; PMI: postmortem interval in hours.

TFEB protein levels (to tubulin) were significantly increased by $61 \%(p<0.01)$ at Braak stage IV AD brains compared to NC (Figure 1). Though Braak stage II also showed increased trend, it was not significantly different from NC brains (Figure 1). On the contrary, nuclear levels of normalized (to lamin) TFEB protein were consistently reduced starting from Braak stage IV (Figure 2). The reduction was $52 \%$ $(p<0.01)$ at Braak stage IV, 67\% $(p<0.01)$ at stage V, and $85 \%(p<0.01)$ at stage VI (Figure 2). Thus, nuclear TFEB is almost completely lost at Braak stage VI. This also suggests that expression levels of nuclear TFEB are inversely proportional to the extent of tau pathology in $\mathrm{AD}$ brains, since Braak staging is based on the extent of tau pathology [38]. Also, alterations in TFEB levels were independent of postmortem interval, sex, or age. Since TFEB protein levels were normalized to that of lamin or tubulin levels, neuronal loss occurring in AD was controlled. Therefore, it is unlikely that reduction in TFEB protein levels in $\mathrm{AD}$ brains is due to neuronal cell loss. 
AD brain: cytosolic TFEB
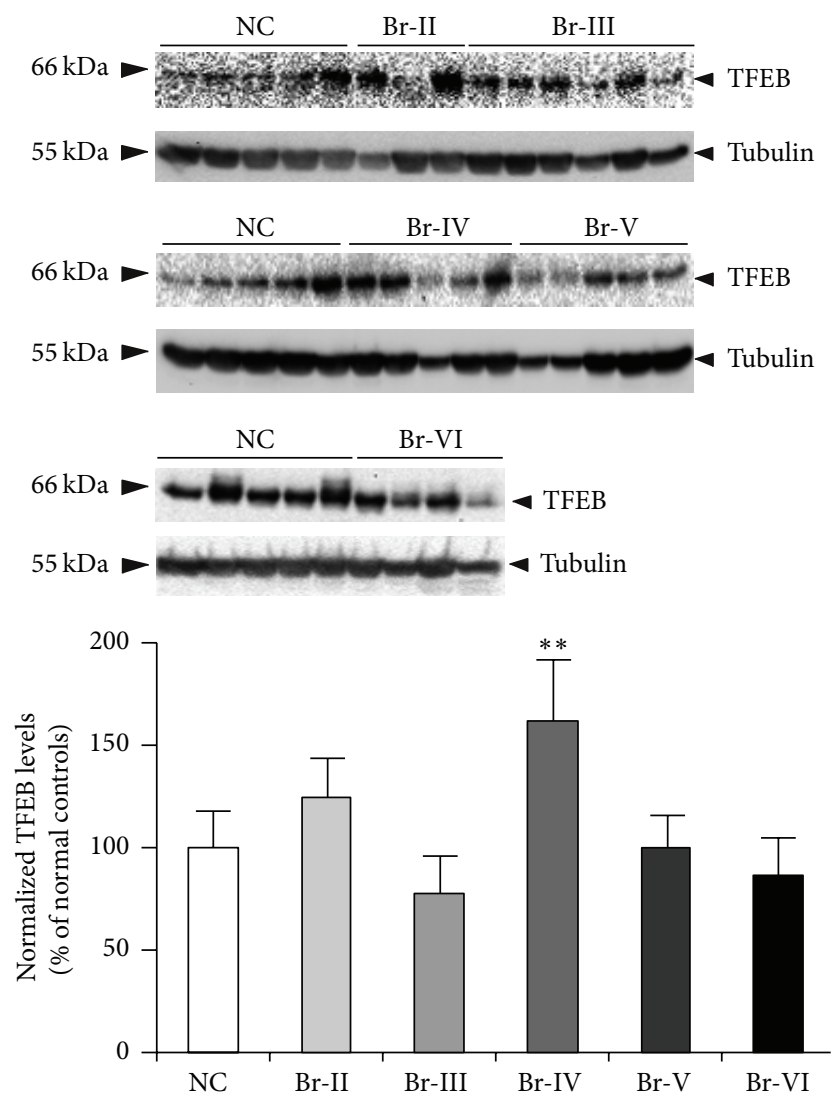

FIGURE 1: TFEB protein levels are not reduced in the cytosolic fractions of $\mathrm{AD}$ brains. Homogenates were prepared from the hippocampus of $\mathrm{AD}$ brains classified as Braak stages II to VI or age-matched normal controls (NC), and cytosolic fractions were separated and subjected to SDS-PAGE. Cytoplasmic fractions showed significantly increased normalized (to tubulin) levels of TFEB protein only at Braak stage IV (61\%). Tubulin was detected to ensure equal loading of samples and as a marker for cytosolic fractions. ${ }^{* *} p<0.01$ by ANOVA followed by Dunnett multiple comparisons test. Data are mean \pm SEM, and " $n$ " are indicated on the figure.

3.2. TFEB Protein Levels Are Significantly Reduced in the Nuclear but Not Cytosolic Fractions of ALS Brains. Since autophagy is also dysregulated in ALS brains [22-24], we obtained ALS and NC brains from NICHD. The demographic details of NC and ALS patients are given in Table 2. The age range of NC subjects was 59-76 years and the PMI was between 3 and $21 \mathrm{~h}$. For ALS patients, the age range was 5987 years and the PMI were 6-22 h. Both males and females were included in the NC and ALS groups. The motor cortices were subjected to homogenization and separated into nuclear and cytoplasmic fractions as described above for AD brains. Immunoblot detection and quantitation of TFEB protein levels in the cytoplasmic fractions did not reveal any changes in the ALS motor cortex compared to NC. However, nuclear fractions showed a $62 \%$ reduction $(p<0.001)$ compared to NC motor cortex (Figure 3 ). Thus, similar to AD brains,
AD brain: nuclear TFEB
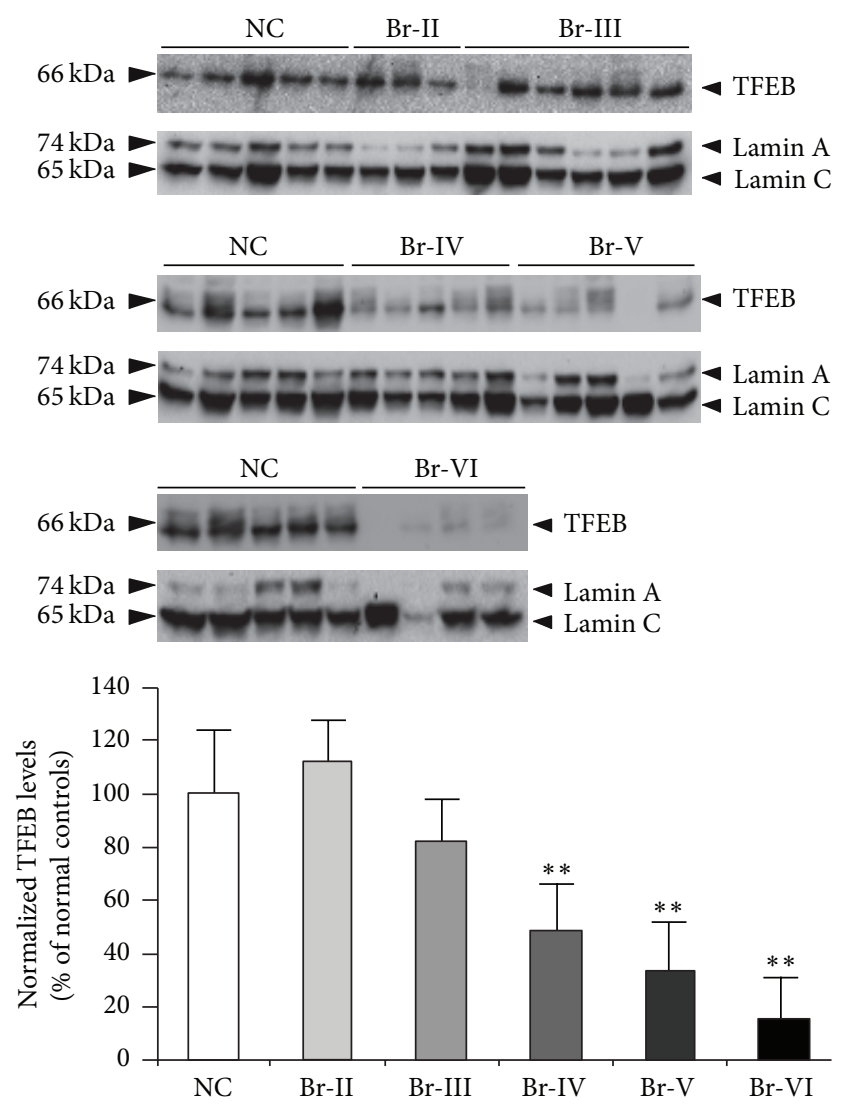

FIGURE 2: Braak-stage-dependent loss of nuclear expression of the transcription factor TFEB in Alzheimer's brains. Homogenates were prepared from the hippocampus of $\mathrm{AD}$ brains classified as Braak stages II to VI or age-matched normal controls (NC) and nuclear fractions were separated and subjected to SDS-PAGE. Nuclear fractions showed robustly decreased normalized (to lamin) TFEB levels starting from Braak stage IV (52\%), stage V (67\%), and stage VI $(85 \%)$. Nuclear lamin was detected as a marker of nuclear fractions. ${ }^{* *} p<0.01$ by ANOVA followed by Dunnett multiple comparisons test. Data are mean \pm SEM, and " $n$ " are indicated on the figure.

reduction in nuclear TFEB levels in ALS brains suggests possible reduction in TFEB's transcriptional activity.

\section{Discussion}

This study provides the first piece of quantitative data on TFEB protein levels in the AD hippocampus staged and diagnosed based on progression of NFT pathology. The results revealed Braak-stage-dependent alterations in the levels of TFEB protein in the $\mathrm{AD}$ brains. This is also the first study to report reduction in TFEB protein levels in the ALS brains. The nuclear fractions of AD revealed striking reductions at later Braak stages, especially showing remarkable reduction at Braak stages V and VI, at which stage there was almost no TFEB protein in the nuclei. On the contrary, TFEB protein levels were increased in the cytosolic fractions at Braak stage IV only, although there was an increased trend at Braak 
TABLE 2: Demographics of normal control subjects and amyotrophic lateral sclerosis (ALS) patients obtained from NICHD Brain and Tissue Bank for Developmental Disorders, used in this study.

\begin{tabular}{lccccc}
\hline Numbers & Diagnosis & Age (y) & Sex & PMI (h) & Tissue \\
\hline 1 & Normal control & 61 & M & 21.00 & Motor cortex \\
2 & Normal control & 59 & M & 10.00 & Motor cortex \\
3 & Normal control & 68 & M & 19.00 & Motor cortex \\
4 & Normal control & 72 & F & 19.00 & Motor cortex \\
5 & Normal control & 76 & F & 3.00 & Motor cortex \\
6 & ALS & 59 & M & 6.00 & Motor cortex \\
7 & ALS & 70 & M & 14.00 & Motor cortex \\
8 & ALS & 71 & F & 6.00 & Motor cortex \\
9 & ALS & 61 & F & 22.00 & Motor cortex \\
10 & ALS & 87 & F & 18.00 & Motor cortex \\
\hline
\end{tabular}

ALS: amyotrophic lateral sclerosis; M: male; F: female; PMI: postmortem interval in hours.

stage II. These results suggest that TFEB protein levels may be selectively lost in the nuclear fractions in a Braak-stagedependent manner.

Braak staging is based on the extent of spread of intracellular deposits of hyperphosphorylated tau protein in NFT, dystrophic neurites, and neuropil threads [38, 39]. At stages I and II, the NFT pathology is restricted to transentorhinal region of the brain with no evidence of cognitive impairments. It appears that at such prodromal disease states TFEB protein levels are not significantly altered as demonstrated in the present study. With the increase in spread of NFT pathology extending to the hippocampus and other limbic areas of the brain at stage IV, TFEB protein levels start showing significant reductions in the nuclear fractions. Since these Braak stages are also accompanied by mild cognitive impairment (MCI), reduction in TFEB protein levels is correlated with the MCI. The most advanced stage of $\mathrm{AD}$ is classified as Braak stages V and VI, by which time the NFT pathology has spread throughout the neocortex and also associated with classical dementia. Reductions in TFEB protein levels in the nuclear fractions were even more robust leading to almost complete absence of TFEB protein at these advanced stages, suggesting that TFEB protein levels are inversely proportional to the extent of NFT pathology and directly correlate with the extent of dementia. Careful analysis also revealed that reduced TFEB in the nuclear fractions is a consequence of more retention of TFEB protein in the soma especially at Braak stage IV. Since no prior study reported TFEB protein levels in AD brains, the present study becomes the first to report and therefore cannot be compared.

In the present study, similar to AD brains, ALS brains also showed marked reductions in nuclear TFEB. TFEB protein levels were also recently shown to be downregulated in both the NSC-34 cell line expressing SOD1 ${ }^{\mathrm{G} 93 \mathrm{~A}}$ mutant protein and spinal motor neurons of transgenic mice expressing SOD $1^{\text {G93A }}$ especially at the later stage of the disease [40], suggesting that SOD $1^{\mathrm{G} 93 \mathrm{~A}}$ mutation affects TFEB expression. Consistent with these findings, TFEB levels were also found
ALS brains: reduced TFEB in the nuclei
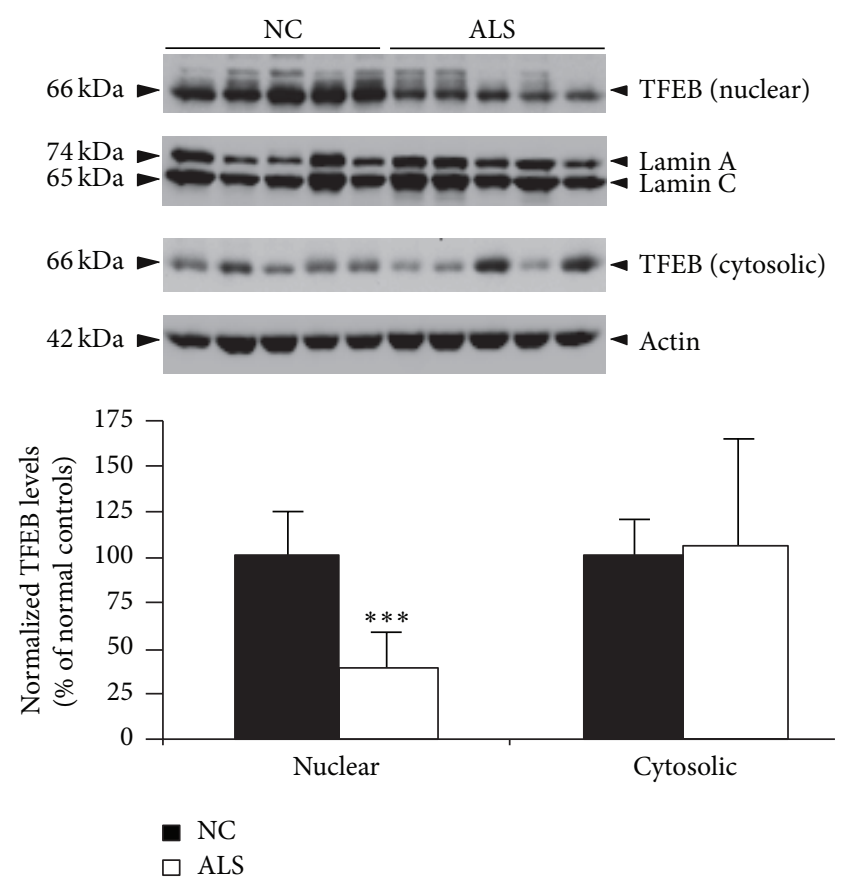

FIGURE 3: Reduced expression of TFEB protein in ALS nuclear fractions. Lysates of patients brains diagnosed with ALS and NC were prepared, and nuclear and cytosolic fractions were separated as in Figures 1 and 2. Actin and lamin were detected as loading controls and used for normalization of TFEB protein levels. Quantitation by ImageJ revealed significantly reduced (62\%) normalized (to lamin) levels of TFEB protein in the nuclear fractions, but no change in the cytosolic fractions. The data are mean \pm SEM. ${ }^{* * *} p<0.001$ by Student's paired $t$-test, $n=5$ per group.

to be reduced in both Huntington's disease cell and mouse models [41], implying that reduced expression of TFEB is a key element in the pathogenesis of not only AD and ALS but also other neurodegenerative diseases.

Since TFEB is a transcription factor, it is physiologically relevant only if it is present in the nucleus. Thus, loss of TFEB in the nuclear fractions indicates that TFEB transcription function in AD and ALS brains is compromised. This conclusion is consistent with the overall evidence that autophagy is reduced in AD brains [18-21] as well as ALS as reflected by accumulation of autophagosomes in the spinal cords of both sporadic and familial cases of ALS [22-24], as well as mouse models of ALS [42-45]. Beclin-1, which plays a key role in inducing autophagy by recruiting membrane to the autophagosomes [46, 47], is also reduced in AD brains [48]. Although another protein, LAMP1, a marker of lysosomes, was shown to be increased in $\mathrm{AD}$ brains as a whole, interestingly, expression of LAMP1 was inversely correlated with hyperphosphorylated tau deposition in individual neurons with tangles [49]. Moreover, though their numbers are not reduced in $\mathrm{AD}$ brains, lysosomes observed in $\mathrm{AD}$ brains are aberrant with discontinuous membranes and irregular shape and size. But since TFEB is a master regulator of lysosome biogenesis and autophagy [28-31], loss of TFEB may be 
primarily responsible for dysregulated autophagy seen in $\mathrm{AD}$ and ALS brains.

Reduced TFEB protein may be responsible for accumulation of NFT and amyloid plaques in AD brains and deposition of a variety of mutant protein aggregates in ALS brains. But how TFEB protein levels per se are reduced is unclear at this time. A recent study demonstrated that, in monocytes derived from sporadic AD patients, upregulation of miR128 correlated with impaired degradation of $\mathrm{A} \beta 42$ [50]. Interestingly, increased miR128 levels led to reduced TFEB levels in blood cells derived from AD patients [50]. Thus, decreased levels of TFEB protein might be responsible for reduced autophagy in these cells which in turn might be responsible for the reduced degradation of $\mathrm{A} \beta 42$. It is also important to note that TFEB expression in the NSC-34 cells increased Beclin-1 and LC3-II protein levels demonstrating that TFEB expression can upregulate autophagy even in a diseased state, which led to increased proliferation and survival [40]. Similarly, more recent studies demonstrated that TFEB overexpression led to marked reduction in the levels of PHFtau [51] and amyloid plaque burden [52]. Further, in the Xlinked SBMA motor neuron disease, caused by block in the autophagosome-lysosome fusion [53], Huntington's disease [54], and Parkinson's disease [55], TFEB could significantly rescue autophagy defects and neurodegeneration. Also, in a recent study, dietary restriction corrected the autophagy defects and partially restored overall neurological symptoms in the dynactin ${ }^{\mathrm{G} 59 \mathrm{~S}}$ mutant model of ALS, most likely through TFEB activation [56]. Collectively, these observations suggest that reduced TFEB levels and autophagy may be responsible for protein accumulations in these disorders and that by restoring TFEB protein levels it may be possible to reduce protein aggregates and thereby reduce neurodegeneration and restore brain function.

\section{Conclusion}

The current study demonstrated a robust reduction in the expression levels of the transcription factor TFEB in the nuclear but not cytosolic fractions of AD and ALS patients' brains relative to normal controls, which may be responsible for the widely reported defective autophagy markers in these disorders. Thus, by restoring TFEB expression, it may be possible to enhance autophagy in these disorders, thereby rescuing neurodegeneration and its associated behavioral deficits.

\section{Competing Interests}

The authors declare no competing interests related to the research covered in this paper.

\section{Acknowledgments}

The authors are grateful to have received Alzheimer's brain tissues provided by the "Harvard Brain Tissue Resource Center," which is supported in part by PHS Grant no. R24MH068855 at the McLean Hospital. They are also grateful to NICHD Brain and Tissue Bank for Developmental Disorders at the University of Maryland, Baltimore, MD, for providing them with ALS and normal control brains.

\section{References}

[1] A. Wimo, B. Winblad, and L. Jönsson, "The worldwide societal costs of dementia: estimates for 2009," Alzheimer's and Dementia, vol. 6, no. 2, pp. 98-103, 2010.

[2] A. Serrano-Pozo, M. P. Frosch, E. Masliah, and B. T. Hyman, "Neuropathological alterations in Alzheimer disease," Cold Spring Harbor Perspectives in Medicine, vol. 1, no. 1, Article ID a006189, 2011.

[3] E. Braak, K. Griffing, K. Arai, J. Bohl, and H. Bratzke, "Neuropathology of Alzheimer's disease. What is new since A. Alzheimer?" European Archives of Psychiatry and Clinical Neurosciences, vol. 249, supplement 3, pp. 14-22, 1999.

[4] M. Gatz, C. A. Reynolds, L. Fratiglioni et al., "Role of genes and environments for explaining Alzheimer disease," Archives of General Psychiatry, vol. 63, no. 2, pp. 168-174, 2006.

[5] M. I. Kamboh, F. Y. Demirci, X. Wang et al., "Genomewide association study of Alzheimer's disease," Translational Psychiatry, vol. 2, article ell7, 2012.

[6] A. Al-Chalabi and O. Hardiman, "The epidemiology of ALS: a conspiracy of genes, environment and time," Nature Reviews Neurology, vol. 9, no. 11, pp. 617-628, 2013.

[7] M. E. Cudkowicz, D. McKenna-Yasek, P. E. Sapp et al., "Epidemiology of mutations in superoxide dismutase in amyotrophic lateral sclerosis," Annals of Neurology, vol. 41, no. 2, pp. 210-221, 1997.

[8] A. E. Renton, A. Chiò, and B. J. Traynor, "State of play in amyotrophic lateral sclerosis genetics," Nature Neuroscience, vol. 17, no. 1, pp. 17-23, 2014.

[9] A. C. Ludolph, C. Bendotti, E. Blaugrund et al., "Guidelines for preclinical animal research in ALS/MND: a consensus meeting," Amyotrophic Lateral Sclerosis, vol. 11, no. 1-2, pp. 3845, 2010.

[10] J. López-Bastida, L. Perestelo-Pérez, F. Montón-Álvarez, P. Serrano-Aguilar, and J. L. Alfonso-Sanchez, "Social economic costs and health-related quality of life in patients with amyotrophic lateral sclerosis in Spain," Amyotrophic Lateral Sclerosis, vol. 10, no. 4, pp. 237-243, 2009.

[11] K. Schepelmann, Y. Winter, A. E. Spottke et al., "Socioeconomic burden of amyotrophic lateral sclerosis, myasthenia gravis and facioscapulohumeral muscular dystrophy," Journal of Neurology, vol. 257, no. 1, pp. 15-23, 2010.

[12] H. Mitsumoto, B. R. Brooks, and V. Silani, "Clinical trials in amyotrophic lateral sclerosis: why so many negative trials and how can trials be improved?" The Lancet Neurology, vol. 13, no. 11, pp. 1127-1138, 2014.

[13] R. G. Miller, J. D. Mitchell, M. Lyon, and D. H. Moore, "Riluzole for amyotrophic lateral sclerosis (ALS)/motor neuron disease (MND)," Cochrane Database of Systematic Reviews, no. 1, Article ID CD001447, 2007.

[14] R. G. Miller, J. D. Mitchell, and D. H. Moore, "Riluzole for amyotrophic lateral sclerosis (ALS)/motor neuron disease (MND)," Cochrane Database of Systematic Reviews, vol. 3, Article ID CD001447, 2012.

[15] M. M. Lipinski, B. Zheng, T. Lu et al., "Genome-wide analysis reveals mechanisms modulating autophagy in normal brain aging and in Alzheimer's disease," Proceedings of the National 
Academy of Sciences of the United States of America, vol. 107, no. 32, pp. 14164-14169, 2010.

[16] A. M. Cuervo, "Autophagy and aging: keeping that old broom working," Trends in Genetics, vol. 24, no. 12, pp. 604-612, 2008.

[17] M. Martinez-Vicente, G. Sovak, and A. M. Cuervo, "Protein degradation and aging," Experimental Gerontology, vol. 40, no. 8-9, pp. 622-633, 2005.

[18] W. H. Yu, A. M. Cuervo, A. Kumar et al., "Macroautophagya novel $\beta$-amyloid peptide-generating pathway activated in Alzheimer's disease," Journal of Cell Biology, vol. 171, no. 1, pp. 87-98, 2005.

[19] B. Boland, A. Kumar, S. Lee et al., "Autophagy induction and autophagosome clearance in neurons: relationship to autophagic pathology in Alzheimer's disease," The Journal of Neuroscience, vol. 28, no. 27, pp. 6926-6937, 2008.

[20] R. A. Nixon, "Autophagy, amyloidogenesis and Alzheimer disease," Journal of Cell Science, vol. 120, part 23, pp. 4081-4091, 2007.

[21] R. A. Nixon, J. Wegiel, A. Kumar et al., "Extensive involvement of autophagy in Alzheimer disease: an immuno-electron microscopy study," Journal of Neuropathology and Experimental Neurology, vol. 64, no. 2, pp. 113-122, 2005.

[22] S. Sasaki, "Autophagy in spinal cord motor neurons in sporadic amyotrophic lateral sclerosis," Journal of Neuropathology and Experimental Neurology, vol. 70, no. 5, pp. 349-359, 2011.

[23] N. F. Bence, R. M. Sampat, and R. R. Kopito, "Impairment of the ubiquitin-proteasome system by protein aggregation," Science, vol. 292, no. 5521, pp. 1552-1555, 2001.

[24] A. Li, X. Zhang, and W. Le, "Altered macroautophagy in the spinal cord of SOD1 mutant mice," Autophagy, vol. 4, no. 3, pp. 290-293, 2008.

[25] B. A. Bahr and J. Bendiske, "The neuropathogenic contributions of lysosomal dysfunction," Journal of Neurochemistry, vol. 83, no. 3, pp. 481-489, 2002.

[26] A. M. Cataldo, D. J. Hamilton, J. L. Barnett, P. A. Paskevich, and R. A. Nixon, "Properties of the endosomal-lysosomal system in the human central nervous system: disturbances mark most neurons in populations at risk to degenerate in Alzheimer's disease," The Journal of Neuroscience, vol. 16, no. 1, pp. 186-199, 1996.

[27] E. Tokuda, T. Brännström, P. M. Andersen, and S. L. Marklund, "Low autophagy capacity implicated in motor system vulnerability to mutant superoxide dismutase," Acta Neuropathologica Communications, vol. 4, article 6, 2016.

[28] M. Sardiello, M. Palmieri, A. di Ronza et al., "A gene network regulating lysosomal biogenesis and function," Science, vol. 325, no. 5939, pp. 473-477, 2009.

[29] C. Settembre, C. Di Malta, V. A. Polito et al., "TFEB links autophagy to lysosomal biogenesis," Science, vol. 332, no. 6036, pp. 1429-1433, 2011.

[30] S. Peña-Llopis, S. Vega-Rubin-De-Celis, J. C. Schwartz et al., "Regulation of TFEB and V-ATPases by mTORC1," EMBO Journal, vol. 30, no. 16, pp. 3242-3258, 2011.

[31] D. L. Medina, A. Fraldi, V. Bouche et al., "Transcriptional activation of lysosomal exocytosis promotes cellular clearance," Developmental Cell, vol. 21, no. 3, pp. 421-430, 2011.

[32] B. Dehay, J. Bové, N. Rodríguez-Muela et al., "Pathogenic lysosomal depletion in Parkinson's disease," Journal of Neuroscience, vol. 30, no. 37, pp. 12535-12544, 2010.

[33] W. A. Dunn Jr., "Autophagy and related mechanisms of lysosome-mediated protein degradation," Trends in Cell Biology, vol. 4, no. 4, pp. 139-143, 1994.
[34] A. Kuma, M. Hatano, M. Matsui et al., "The role of autophagy during the early neonatal starvation period," Nature, vol. 432, no. 7020, pp. 1032-1036, 2004.

[35] D. J. Klionsky and S. D. Emr, "Autophagy as a regulated pathway of cellular degradation," Science, vol. 290, no. 5497, pp. 1717-1721, 2000.

[36] M. K. Lakshmana, J. Y. Chung, S. Wickramarachchi et al., "A fragment of the scaffolding protein RanBP9 is increased in Alzheimer's disease brains and strongly potentiates amyloid- $\beta$ peptide generation," The FASEB Journal, vol. 24, no. 1, pp. 119127,2010

[37] C. Settembre, R. Zoncu, D. L. Medina et al., "A lysosometo-nucleus signalling mechanism senses and regulates the lysosome via mTOR and TFEB," The EMBO Journal, vol. 31, no. 5, pp. 1095-1108, 2012.

[38] H. Braak and E. Braak, "Neuropathological stageing of Alzheimer-related changes," Acta Neuropathologica, vol. 82, no. 4, pp. 239-259, 1991.

[39] H. Braak, I. Alafuzoff, T. Arzberger, H. Kretzschmar, and K. Tredici, "Staging of Alzheimer disease-associated neurofibrillary pathology using paraffin sections and immunocytochemistry," Acta Neuropathologica, vol. 112, no. 4, pp. 389-404, 2006.

[40] Y. Chen, H. Liu, Y. Guan et al., “The altered autophagy mediated by TFEB in animal and cell models of amyotrophic lateral sclerosis," American Journal of Translational Research, vol. 7, no. 9, pp. 1574-1587, 2015.

[41] A. R. La Spada, "PPARGC1A/PGC- $1 \alpha$, TFEB and enhanced proteostasis in Huntington disease: defining regulatory linkages between energy production and protein-organelle quality control," Autophagy, vol. 8, no. 12, pp. 1845-1847, 2012.

[42] N. Morimoto, M. Nagai, Y. Ohta et al., "Increased autophagy in transgenic mice with a G93A mutant SOD1 gene," Brain Research, vol. 1167, no. 1, pp. 112-117, 2007.

[43] F. Tian, N. Morimoto, W. Liu et al., "In vivo optical imaging of motor neuron autophagy in a mouse model of amyotrophic lateral sclerosis," Autophagy, vol. 7, no. 9, pp. 985-992, 2011.

[44] F. M. Laird, M. H. Farah, S. Ackerley et al., "Motor neuron disease occurring in a mutant dynactin mouse model is characterized by defects in vesicular trafficking," Journal of Neuroscience, vol. 28, no. 9, pp. 1997-2005, 2008.

[45] T. Kabuta, Y. Suzuki, and K. Wada, "Degradation of amyotrophic lateral sclerosis-linked mutant $\mathrm{Cu}, \mathrm{Zn}$-superoxide dismutase proteins by macroautophagy and the proteasome," The Journal of Biological Chemistry, vol. 281, no. 41, pp. 30524-30533, 2006.

[46] X. H. Liang, S. Jackson, M. Seaman et al., "Induction of autophagy and inhibition of tumorigenesis by beclin 1," Nature, vol. 402, no. 6762, pp. 672-676, 1999.

[47] X. Qu, J. Yu, G. Bhagat et al., "Promotion of tumorigenesis by heterozygous disruption of the beclin 1 autophagy gene," Journal of Clinical Investigation, vol. 112, no. 12, pp. 1809-1820, 2003.

[48] F. Pickford, E. Masliah, M. Britschgi et al., "The autophagyrelated protein beclin 1 shows reduced expression in early Alzheimer disease and regulates amyloid $\beta$ accumulation in mice," The Journal of Clinical Investigation, vol. 118, no. 6, pp. 2190-2199, 2008.

[49] M. Barrachina, T. Maes, C. Buesa, and I. Ferrer, "Lysosomeassociated membrane protein 1 (LAMP-1) in Alzheimer's disease," Neuropathology and Applied Neurobiology, vol. 32, no. 5, pp. 505-516, 2006. 
[50] R. Tiribuzi, L. Crispoltoni, S. Porcellati et al., "MiR128 upregulation correlates with impaired amyloid $\beta(1-42)$ degradation in monocytes from patients with sporadic Alzheimer's disease," Neurobiology of Aging, vol. 35, no. 2, pp. 345-356, 2014.

[51] V. A. Polito, H. Li, H. Martini-Stoica et al., "Selective clearance of aberrant tau proteins and rescue of neurotoxicity by transcription factor EB," EMBO Molecular Medicine, vol. 6, no. 9, pp. 1142-1160, 2014.

[52] Q. Xiao, P. Yan, X. Ma et al., "Enhancing astrocytic lysosome biogenesis facilitates $\mathrm{A} \beta$ clearance and attenuates amyloid plaque pathogenesis," Journal of Neuroscience, vol. 34, no. 29, pp. 9607-9620, 2014.

[53] C. J. Cortes, H. C. Miranda, H. Frankowski et al., "Polyglutamine-expanded androgen receptor interferes with TFEB to elicit autophagy defects in SBMA," Nature Neuroscience, vol. 17, pp. 1180-1189, 2014.

[54] T. Tsunemi, T. D. Ashe, B. E. Morrison et al., "PGC-1a rescues Huntington's disease proteotoxicity by preventing oxidative stress and promoting TFEB function," Science Translational Medicine, vol. 4, no. 142, Article ID 142ra97, 2012.

[55] M. Decressac, B. Mattsson, P. Weikop, M. Lundblad, J. Jakobsson, and A. Björklund, "TFEB-mediated autophagy rescues midbrain dopamine neurons from $\alpha$-synuclein toxicity," Proceedings of the National Academy of Sciences of the United States of America, vol. 110, no. 19, pp. E1817-E1826, 2013.

[56] D. Wiesner, J. Sinniger, A. Henriques et al., "Low dietary protein content alleviates motor symptoms in mice with mutant dynactin/dynein-mediated neurodegeneration," Human Molecular Genetics, vol. 24, no. 8, pp. 2228-2240, 2015. 

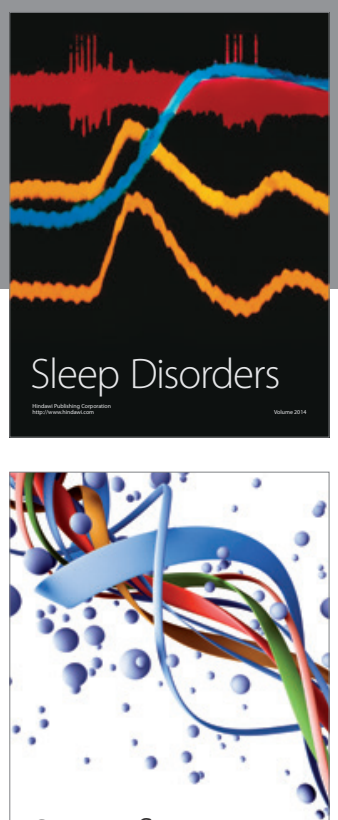

Scientifica
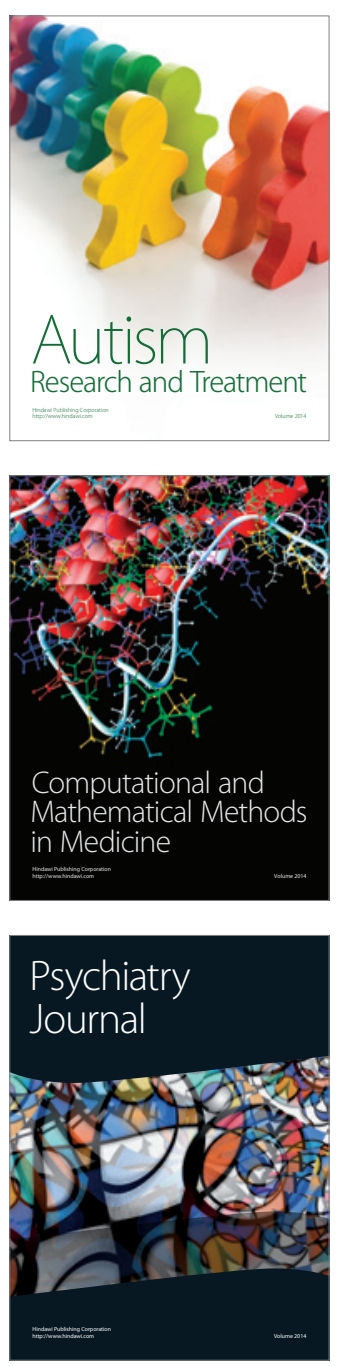
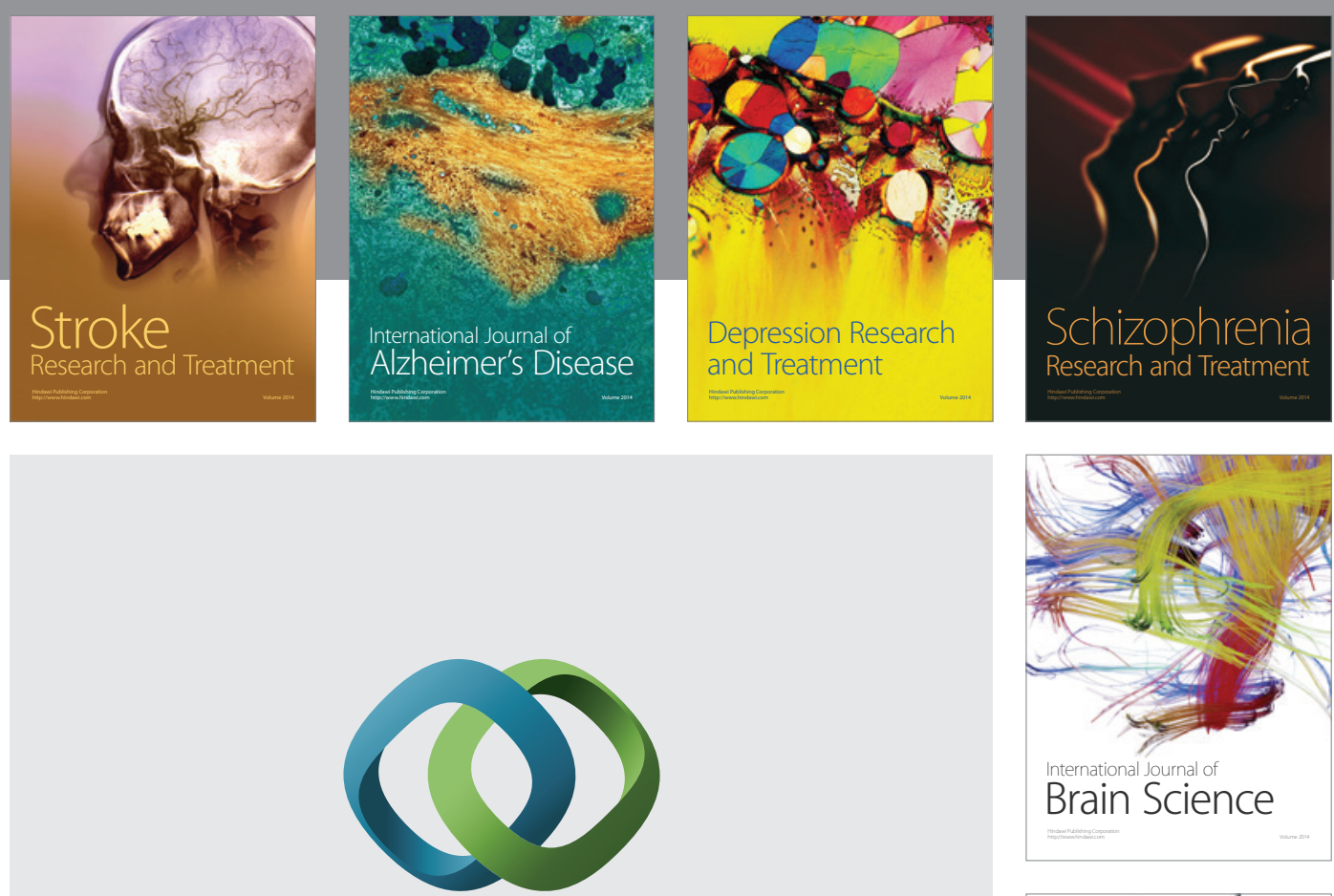

\section{Hindawi}

Submit your manuscripts at

http://www.hindawi.com
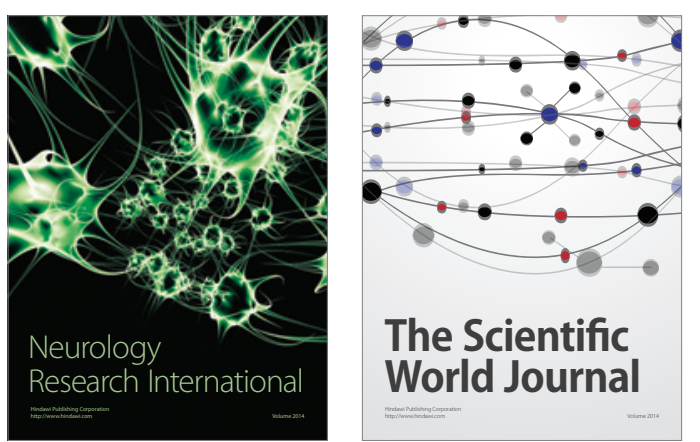

The Scientific World Journal

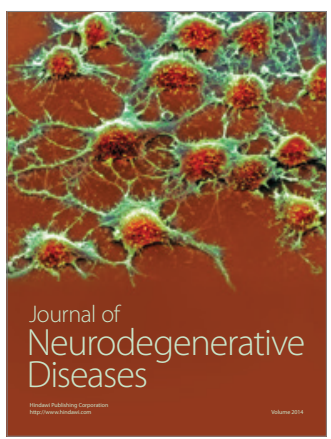

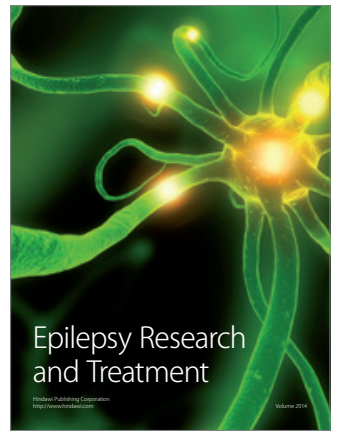

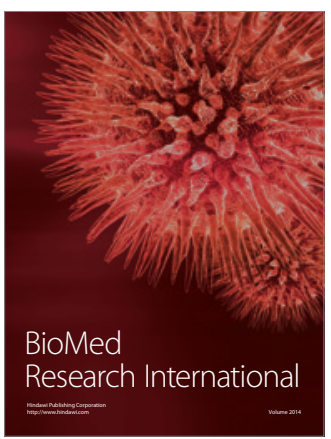

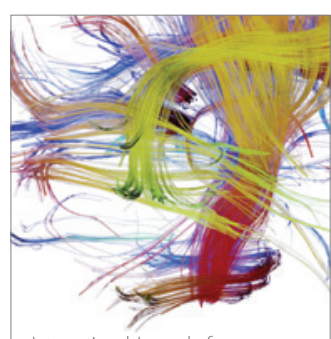

Brain Science

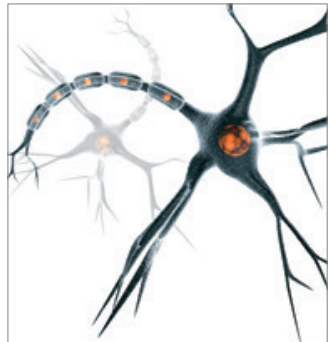

Neural Plasticity
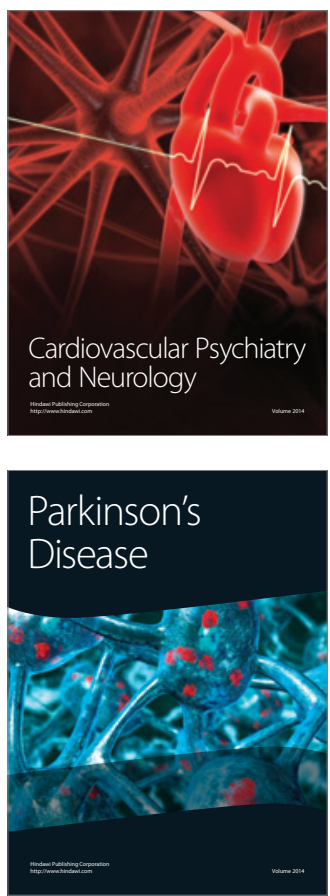